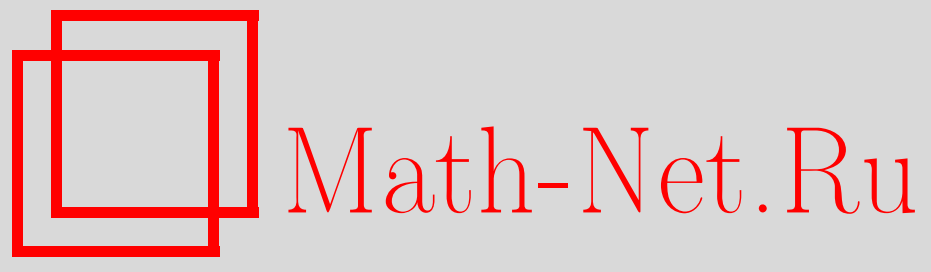

В. А. Рубаков, Фантом без патологий в ультрафиолетовой области, ТМФ, 2006, том 149, номер 3, 409-426

DOI: https://doi.org/10.4213/tmf5532

Использование Общероссийского математического портала Math-Net.Ru подразумевает, что вы прочитали и согласны с пользовательским соглашением http://www . mathnet.ru/rus/agreement

Параметры загрузки:

IP : 54.89 .56 .158

26 апреля 2023 г., 03:03:49

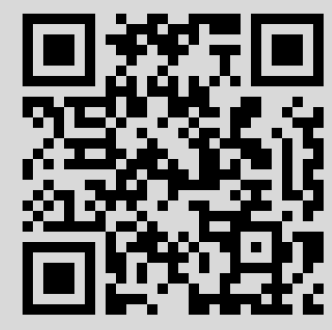




\title{
ФАНТОМ БЕЗ ПАТОЛОГИЙ В УЛЬТРАФИОЛЕТОВОЙ ОБЛАСТИ
}

\begin{abstract}
Представлена простая модель, в которой условие светоподобной доминантности нарушается для пространственно однородных медленно эволюционирующих полей. Флуктуации в пространстве Минковского во внешнем поле с нарушенной лоренц-инвариантностью не содержат духов, тахионов или сверхсветовых мод при пространственных импульсах, изменяющихся в диапазоне от некоторого малого масштаба $\epsilon$ до масштаба ультрафиолетового обрезания, в то время как тахионы и, возможно, духи существуют при трехмерных импульсах, меньших $\epsilon$. Показано, что в отсутствие другой материи возможен космологический режим медленного качения; в этом режиме нарушается условие светоподобной энергодоминантности, но однородность и изотропность еще не полностью разрушены (за счет тонкой настройки), поскольку для фиксированного конформного импульса тахионная мода растет лишь на достаточно коротком временно́м интервале.
\end{abstract}

Ключевые слова: лоренц-инвариантность, векторное поле, космологическое расширение, условие светоподобной энергодоминантности.

\section{1. ВВЕДЕНИЕ}

Наблюдаемый рост интереса к возможным экзотическим гравитационнным явлениям на космологических расстояниях вызван частично наблюдением ускоряющегося расширения Вселенной, частично - развитием теории. Имеющиеся в этой области подходы включают в себя изменение гравитации на сверхбольших масштабах [1], [2] и введение новых легких полей. Среди последних фантомная энергия с уравнением состояния $p+\rho<0$, нарушающая таким образом условие светоподобной доминантности, представляет особый интерес как с чисто теоретической точки зрения, так, возможно, и для космологии [3]. Если бы фантом доминировал в течение поздней космологической эволюции, потенциально наблюдаемым свойством было бы ускоряющееся ускорение Вселенной ${ }^{1)}$; если бы фантом отвечал за инфляциею, спектр реликтовых гравитационных волн был бы голубым, что также потенциально возможно

\footnotetext{
1)Этот эффект можно имитировать в скалярно-тензорных теориях гравитации [4] с материей, удовлетворяющей неравенству $p+\rho>0$, см., однако, [5].
}

* Институт ядерных исследований РАН, Москва, Россия. E-mail: rubakov@ms2.inr.ac.ru 
наблюдать. Однако большинство построенных к настоящему моменту теорий поля, нарушающих условие светоподобной энергодоминантности, страдают патологиями в ультрафиолетовой (УФ) области (здесь и далее под УФ-областью мы будем понимать энергии достаточно высокие, но не превосходящие масштаб УФ-обрезания в эффективной теории поля): они содержат либо духи, либо тахионы, либо распространяющиеся со сверхсветовой скоростью моды, либо комбинации всего этого. Более того, существуют аргументы общего характера, из которых следует, что в размерности четыре любая ${ }^{2)}$ такая теория содержит УФ-нестабильности [6], [7]. Тем не менее чисто феноменологический анализ [8] показывает, что подобная ситуация не обязательно реализуется в общем случае. Кроме того, модель Двали-ГабададзеПоррати мира на бране [2], которая может обладать хорошим поведением в УФобласти [9], допускает период ускоренного космологического расширения, когда эффективно уравнение состояния соответствует $p+\rho<0$ [10]. Таким образом, имеют смысл попытки построения примера четырехмерного теоретико-полевого фантома с непатологическим УФ-поведением.

Согласно аргументам работы [6] весьма маловероятно, что существуют вообще не имеющие патологий теории поля, в которых $p+\rho<0$. В качестве умеренного подхода мы можем начать с рассмотрения теории поля в пространстве Минковского, являющейся самосогласованной на всех энергетических масштабах от нуля до масштаба УФ-обрезания $\mathcal{M}$ в эффективной теории. Затем мы деформируем эту теорию в инфракрасной (ИК) области так, чтобы ее поведение при высоких энергиях не ухудшилось, однако при этом условие светоподобной энергодоминантности нарушалось бы для пространственно однородных конфигураций, а патологические состояния появлялись бы только при энергиях ниже некоторого малого масштаба $\epsilon^{3)}$. Если следовать этому подходу, необходимо принять во внимание, что возможны сверхсветовые моды, которые могут возникать для больших значений импульсов после модификации теории в ИК-области: этот эффект, даже при небольшой его величине, указывал бы на несамосогласованность всей теории [12].

В космологическом контексте теория такого типа может быть приемлемой, если энергетический масштаб $\epsilon$ находится ниже или по крайней мере достаточно близко к хаббловскому масштабу. Следует ожидать, что последнее свойство требует тонкой настройки параметров помимо и сверх других настроек, необходимых для того, чтобы сделать теорию не противоречащей наблюдениям.

Один из путей осуществления такого подхода состоит в том, чтобы начать с более или менее обычной теории с двумя производными и правильным поведением ниже масштаба $\mathcal{M}$, по крайней мере для некоторых внешних полей, а затем добавить к лагранжиану член с одной производной, умноженный на малый параметр $\epsilon$. Такое

\footnotetext{
2) Возможное исключение относится к (спонтанному) нарушению трехмерной вращательной инвариантности [6].

3) В теориях с духами и ненарушенной лоренцевой симметрией, такой подход не работает, поскольку для всех пространственных импульсов и частот будут возникать нестабильности. Однако коль скоро лоренцева инвариантность нарушена, это рассуждение более неприменимо, и содержащая духи теория может оказаться жизнеспособной даже для достаточно больших ИК масштабов [11].
} 
построение сложно реализовать в скалярных теориях, и это естественным образом приводит к рассмотрению теорий, содержащих векторное поле (поля). К настоящему времени стало понятно, что можно избежать связанных с УФ-областью проблем, присущих векторным теориям без калибровочной инвариантности [13], и что члены с одной производной действительно порождают интересную ИК-динамику [14].

В настоящей работе на основании приведенных выше соображений мы построили простую модель. Эта модель описывается в разделе 2. Предполагается, что фоновые поля нарушают лоренцеву инвариантность, а трехмерная вращательная инвариантность остается ненарушенной. В разделе 3 мы проверяем, что в плоском пространстве-времени при значениях трехмерных импульсов $q$ выше критического импульса $q_{\mathrm{c}} \sim \epsilon$ не имеется духов, тахионов или сверхсветовых мод. При $q<q_{\mathrm{c}}$ имеются тахионы и, возможно, духи.

Обращаясь в разделе 4 к космологической эволюции, мы показываем, что в отсутствие другой материи при подходящем выборе потенциала возможен режим медленного качения, коль скоро однородные поля, заполняющие Вселенную, принимают подходящие значения. В таком режиме уравнение состояния отвечает случаю $p+\rho<0$, причем $|p+\rho| \ll \rho$, и, действительно, поля медленно закатываются вверx по потенциалу. В рассматриваемой нами модели режим подобного медленного качения возникает при $H \ll \epsilon$, так что в течение некоторого периода времени тахионная мода является подгоризонтной.

Затем мы исследуем неоднородные возмущения полей около медленно катящегося фона (раздел 5). Мы показываем, что для фиксированного конформного импульса $k$ опасная тахионная мода растет в течение конечного промежутка времени, до тех пор пока не станет надгоризонтной. Надгоризонтные моды не растут; некоторые из них, в том числе бывшие тахионные моды, вморожены (как в случае минимального скалярного поля в ускоряющейся Вселенной), некоторые уменьшаются по амплитуде. Для бывшей тахионной моды это свойство не вполне тривиально и связано с тем, что эта мода является бесщелевой в пространстве Минковского. С помощью тонкой настройки время роста тахионной моды можно сделать достаточно малым, и это приведет к тому, что модель станет жизнеспособной. В разделе 6 мы подводим итоги.

\section{2. МОДЕЛЬ}

Модель, которую мы хотели бы представить, имеет кинетические члены с двумя производными, аналогичные таковым из работы [13], член с одной производной, напоминающий выражение из работы [14], а также потенциальный член. В модели имеется одно векторное поле $B_{\mu}$ и одно скалярное поле $\Phi$. Лагранжиан имеет вид ${ }^{4}$

$$
L=L^{(2)}+L^{(1)}+L^{(0)}
$$

где верхними индексами обозначено число производных, и мы выбираем

$$
L^{(2)}=-\frac{1}{2} \alpha(\xi) g^{\nu \lambda} D_{\mu} B_{\nu} D^{\mu} B_{\lambda}+\frac{1}{2} \beta(\xi) D_{\mu} B_{\nu} D^{\mu} B_{\lambda} \cdot \frac{B^{\nu} B^{\lambda}}{\mathcal{M}^{2}}+\frac{1}{2} \partial_{\mu} \Phi \partial^{\mu} \Phi,
$$

\footnotetext{
4) Пространственно-временная сигнатура имеет вид $(+,-,-,-)$.
} 


$$
\begin{gathered}
L^{(1)}=\epsilon \partial_{\mu} \Phi B^{\mu}, \\
L^{(0)}=-V(B, \Phi),
\end{gathered}
$$

где $D_{\mu} B_{\nu}=\partial_{\mu} B_{\nu}-\Gamma_{\mu \nu}^{\lambda} B_{\lambda}-$ ковариантная производная, $\xi=B_{\mu} B^{\mu} / \mathcal{M}^{2}$. Здесь $\alpha$ и $\beta$ - функции порядка единицы, а $\epsilon$ - свободный положительный параметр (ИКмасштаб); $\mathcal{M}$ можно рассматривать как масштаб УФ-обрезания в эффективной теории, так что $\mathcal{M} \gg \epsilon$. Мы будем рассматривать фон с нарушением лоренцевой инвариантности, для которого $\xi \neq 0$.

Лагранжиан является лоренц-инвариантным, если $V$ зависит от $X=\sqrt{B_{\mu} B^{\mu}}$. Для простоты мы главным образом будем рассматривать потенциалы вида

$$
V=U(X)+W(\phi)
$$

Начнем с пространства Минковского и рассмотрим статический однородный фон

$$
B_{0}=X=\text { const }, \quad B_{i}=0, \quad \Phi=\phi=\text { const },
$$

где $B_{0}$ и $B_{i}$ - временна́я и пространственная компоненты $B_{\mu}$. Мы будем рассматривать малые неоднородные возмущения $b_{0}, b_{i}, \varphi$ такие, что

$$
B_{0}=X+b_{0}, \quad B_{i}=b_{i}, \quad \Phi=\phi+\varphi .
$$

Их массовые члены имеют вид

$$
-\frac{m_{0}^{2}}{2} b_{0}^{2}-\frac{m_{1}^{2}}{2} b_{i}^{2}-\frac{m_{\varphi}^{2}}{2} \varphi^{2},
$$

где

$$
\begin{aligned}
& m_{0}^{2}=U_{X X}, \\
& m_{1}^{2}=-\frac{1}{X} U_{X}, \\
& m_{\varphi}^{2}=W_{\phi \phi} .
\end{aligned}
$$

Здесь мы ввели удобные обозначения для производных:

$$
\frac{\partial U}{\partial X} \equiv U_{X}, \quad \frac{\partial^{2} U}{\partial X^{2}} \equiv U_{X X} \quad \text { и т.д. }
$$

Сделаем замечание относительно тензора энергии-импульса. Для конфигурации поля в случае $B_{i}=0$ нётеровский тензор энергии-импульса имеет вид

$$
T_{\mu \nu}=\kappa \partial_{\mu} B_{0} \partial_{\nu} B_{0}+\partial_{\mu} \Phi \partial_{\nu} \Phi+\delta_{\mu 0} \epsilon \partial_{\nu} \Phi B_{0}-\eta_{\mu \nu} L
$$

где

$$
\kappa(X)=\frac{X^{2}}{\mathcal{M}^{2}} \beta(X)-\alpha(X) .
$$


Ниже мы полагаем, что $\kappa>0$. Если $B_{0}$ и $\Phi$ пространственно однородны, $B_{0}=X(t)$, $\Phi=\phi(t)$, то плотность энергии и давление имеют вид

$$
\begin{aligned}
& \rho=\frac{\kappa}{2} \dot{X}^{2}+\frac{1}{2} \dot{\phi}^{2}+V, \\
& p=\frac{\kappa}{2} \dot{X}^{2}+\frac{1}{2} \dot{\phi}^{2}+\epsilon \dot{\phi} X-V .
\end{aligned}
$$

Соотношение $p+\rho<0$ выполнено, если временнь́е производные полей малы и

$$
\dot{\phi} X<0 \text {. }
$$

В космологическом контексте временнь́е производные действительно будут малы, если поля медленно катятся вверх по потенциалу; далее мы увидим, как это требование вместе с соотношением (13) может выполняться.

\section{3. СПЕКТР В ПРОСТРАНСТВЕ МИНКОВСКОГО}

3.1. Безмассовый случай. Временно не будем принимать во внимание эффекты, связанные с гравитацией, и рассмотрим спектр нашей модели в пространстве Минковского в случае фона (6). Начнем с того, что пренебрежем массовыми членами и запишем квадратичный лагранжиан для возмущений:

$$
L_{b_{i}, b_{0}, \varphi}=\frac{\alpha}{2} \partial_{\mu} b_{i} \partial^{\mu} b_{i}+\frac{\kappa}{2} \partial_{\mu} b_{0} \partial^{\mu} b_{0}+\frac{1}{2} \partial_{\mu} \varphi \partial^{\mu} \varphi+\epsilon \partial_{0} \varphi b_{0}-\epsilon \partial_{i} \varphi b_{i}
$$

Из этого лагранжиана можно сразу же найти спектр. Поперечные компоненты $b_{i}$ отщепляются и удовлетворяют стандартному безмассовому дисперсионному соотношению $\omega^{2}=q^{2}$. Возмущение $b_{\mathrm{L}}$, определяющее продольную часть вектора $b_{i}$ в соответствии с $b_{i}=\left(q_{i} / q\right) b_{\mathrm{L}}$, смешивается с $b_{0}$ и $\varphi$. Среди трех мод - линейных комбинаций $b_{\mathrm{L}}, b_{0}$ и $\varphi$ - существует одна мода, для которой $\varphi=0, b_{0}=b_{\mathrm{L}}$, с таким же дисперсионным соотношением $\omega^{2}=q^{2}$ и две моды с дисперсионными соотношениями

$$
\omega^{2}=q^{2}+\frac{\epsilon^{2}}{2 \kappa} \pm \sqrt{\left(\frac{1}{\alpha}+\frac{1}{\kappa}\right) \epsilon^{2} q^{2}+\frac{\epsilon^{4}}{4 \kappa^{2}}} .
$$

Эти моды не являются духами ни при каких q. Рассмотрим их свойства для двух различных областей значений импульсов.

Начнем с больших значений импульсов, полагая, что $q^{2} \gg\left(\alpha^{-1}+\kappa^{-1}\right) \epsilon^{2}$. Разлагая по обратному импульсу, получаем

$$
\omega=q \pm \frac{1}{2} \epsilon \sqrt{\frac{1}{\alpha}+\frac{1}{\kappa}}+\frac{1}{8} \frac{\epsilon^{2}}{q}\left(\frac{1}{\kappa}-\frac{1}{\alpha}\right) .
$$

Мы видим, что моды имеют групповую скорость $\partial \omega / \partial q$, меньшую, чем скорость света, если

$$
\alpha>\kappa
$$

В дальнейшем мы будем рассматривать именно этот случай. 
Теперь обратимся к области малых импульсов. Существует критический импульс

$$
q_{\mathrm{c}}^{2}=\frac{\epsilon^{2}}{\alpha}
$$

Выше этого значения импульса тахионных мод нет. При $q^{2}<q_{\mathrm{c}}^{2}$ одна из мод (которой соответствует знак минус в выражении (15)) является тахионной. Наибольшее возможное тахионное значение $\left|\omega^{2}\right|$ достигается при

$$
q^{2}=\frac{\epsilon^{2}}{4 \alpha} \frac{2 \alpha+\kappa}{\alpha+\kappa},
$$

и для такого импульса мы имеем

$$
\omega_{\min }^{2}=-\frac{\kappa}{4(\alpha+\kappa)} \frac{\epsilon^{2}}{\alpha} .
$$

Стоит отметить, что это значение намного меньше, чем квадрат максимального тахионного импульса (18), если

$$
\alpha \gg \kappa,
$$

что, конечно же, требует настройки параметров.

Таким образом, безмассовая модель на фоне пространства Минковского в области больших импульсов не имеет сверхсветовых, духовых или тахионных мод, а в области малых импульсов имеет тахионные моды.

3.2. Ненулевые массы. Теперь "включим" массы рассматриваемых возмущений, т.е. добавим члены (8) в лагранжиан (14). Ниже мы будем рассматривать случай

$$
m_{\varphi}^{2}=0, \quad m_{0}^{2} \sim m_{1}^{2}, \quad \epsilon^{2} \gg m_{0}^{2}, m_{1}^{2} .
$$

Более того, для простоты мы предположим, что выполнено соотношение (20).

Поперечные моды $b_{i}$ снова отщепляются; дисперсионное соотношение для них имеет вид

$$
\omega_{\text {transv }}^{2}=q^{2}+\frac{m_{1}^{2}}{\alpha}
$$

Уравнения для $b_{0}, \varphi$ и $b_{\mathrm{L}}$ таковы:

$$
\begin{aligned}
& \left(\omega^{2}-q^{2}-\frac{m_{0}^{2}}{\kappa}\right) b_{0}+i \frac{\epsilon}{\kappa} \omega \varphi=0 \\
& \left(\omega^{2}-q^{2}-\frac{m_{1}^{2}}{\alpha}\right) b_{\mathrm{L}}-i \frac{\epsilon}{\alpha} q \varphi=0 \\
& \left(\omega^{2}-q^{2}\right) \varphi-i \epsilon \omega b_{0}+i \epsilon q b_{\mathrm{L}}=0
\end{aligned}
$$

Анализ мод для случая больших импульсов аналогичен проведенному выше. Разлагая $\omega$ по обратному импульсу, получаем в рамках наших допущений, что две моды (16) остаются такими же (с точностью до малых поправок), а третья мода изменяется следующим образом:

$$
\omega=q+\frac{m_{0}^{2}+m_{1}^{2}}{\alpha+\kappa} \frac{1}{2 p} .
$$


При положительных $m_{0}^{2}$ и $m_{1}^{2}$ эта мода также является субсветовой. Все моды при больших импульсах содержат кинетические члены правильного знака, поэтому в УФ-области духов нет.

Теперь рассмотрим моды, соответствующие малым импульсам, и предположим, что $m_{0}^{2}>0, m_{1}^{2}>0$ (на случае $m_{0}^{2}<0, m_{1}^{2}>0$ мы остановимся в конце данного раздела). Сразу видно, что тахионная мода снова существует при $q^{2}<q_{\mathrm{c}}^{2}$, а наинизшее значение величины $\omega^{2}$ для тахионной моды по-прежнему определяется выражением (19). Поучительно исследовать дисперсионные соотношения при очень малых значениях импульсов, а именно при $q^{2} \ll m_{1}^{2} / \alpha$. Анализируя уравнения (23), находим, что одна мода содержит главным образом поле $b_{\mathrm{L}}$ и имеет дисперсионное соотношение

$$
\omega_{b_{\mathrm{L}}}^{2}=\frac{m_{1}^{2}}{\alpha}
$$

Имеются еще две моды: одна нетахионная мода с дисперсионным соотношением

$$
\omega^{2}=\frac{\epsilon^{2}}{\kappa}
$$

и одна тахионная мода с дисперсионным соотношением

$$
\omega_{\text {tach }}^{2}=-\frac{m_{0}^{2}}{m_{1}^{2}} q^{2} .
$$

Ни одна из мод с положительными $\omega^{2}$ не является духом. Важно, что $\omega^{2}$ для тахионной моды стремится к нулю при $q^{2} \rightarrow 0$; действительно, хотя формула (27) буквально справедлива только при $\epsilon^{2} \gg m_{0}^{2}, m_{1}^{2}$, отсутствие щели в тахионной моде, т.е. свойство $\omega_{\text {tach }}^{2} \propto-q^{2}$ при $q^{2} \rightarrow 0$, справедливо в общем случае.

В завершение этого раздела остановимся на случае

$$
m_{0}^{2}<0, \quad m_{1}^{2}>0
$$

по-прежнему полагая, что соотношения (21) выполнены для абсолютных значений. Из (16) и (24) следует, что в этом случае при больших импульсах все моды попрежнему являются субсветовыми, если

$$
\left|m_{0}^{2}\right|<m_{1}^{2}
$$

Тахионная мода снова появляется при $q^{2}=q_{\mathrm{c}}^{2}$. Особое свойство, характерное для данного случая, состоит в том, что при малых импульсах, точнее, при $q^{2}<\left|m_{0}^{2}\right| / \kappa$, эта мода снова становится нетахионной, и возникает состояние с отрицательной энергией - дух в спектре. Рассмотрим, например, специальный случай

$$
m_{0}^{2}=-m_{1}^{2}<0
$$


В этом случае тахион существует в конечном интервале импульсов ${ }^{5)}$, а именно при $m_{1}^{2} / \kappa<q^{2}<\left(\epsilon^{2}-m_{1}^{2}\right) / \alpha$. При всех значениях импульсов существует мода с безмассовым дисперсионным соотношением

$$
\omega^{2}=q^{2}
$$

а также моды (16), которые являются субсветовыми при больших импульсах. При больших $q^{2}$ мода (29) имеет положительную энергию, в то время как точно при том же значении импульса, при котором тахион исчезает, т.е. при $q^{2}=m_{1}^{2} / \kappa$, эта мода становится духом. Она остается духом при всех значениях $q^{2}<m_{1}^{2} / \kappa$.

\section{4. КОСМОЛОГИЧЕСКАЯ ЭВОЛЮЦИЯ}

4.1. Полевые уравнения. В случае пространственно однородных полей с $B_{i}=0$ в пространственно-плоской метрике Фридмана-Робертсона-Уокера

$$
d s^{2}=N^{2}(t) d t^{2}-a^{2}(t) d \mathbf{x}^{2}
$$

лагранжиан (1) задается формулой

$$
\sqrt{g} L=\frac{\kappa}{2} \frac{a^{3}}{N} \dot{X}^{2}-\frac{3 \alpha}{2} \frac{\dot{a}^{2} a}{N} X^{2}+\frac{1}{2} \frac{a^{3}}{N} \dot{\phi}^{2}+\epsilon a^{3} \dot{\phi} X-a^{3} N V(X, \phi),
$$

где $X=N^{-1} B_{0}$. Стоит отметить, что с точностью до зависящего от $X$ множителя второе слагаемое в этом выражении пропорционально гравитационному лагранжиану $\sqrt{-g} R \propto\left(\dot{a}^{2} a\right) / N$ по модулю полной производной. Поэтому эффективная "космологическая" масса Планка, входящая в уравнение Фридмана, модифицируется следующим образом (ср. [15]):

$$
M_{\mathrm{Pl}}^{2} \rightarrow M_{\mathrm{Pl}}^{2}+\frac{\alpha}{4 \pi} X^{2}
$$

Нам надо будет в дальнейшем удостовериться, что это изменение мало ${ }^{6}$.

Из лагранжиана (30) получаем выражения для плотности энергии и давления:

$$
\begin{aligned}
& \rho=-\frac{1}{a^{3}}\left[\frac{\delta S_{X, \phi}}{\delta N}\right]_{N=1}=\frac{\kappa}{2} \dot{X}^{2}-\frac{3 \alpha}{2} H^{2} X^{2}+\frac{1}{2} \dot{\phi}^{2}+V, \\
& p=\frac{1}{3 a^{2}}\left[\frac{\delta S_{X, \phi}}{\delta a}\right]_{N=1}=\frac{\kappa}{2} \dot{X}^{2}+\frac{3 \alpha}{2} H^{2} X^{2}+\alpha \dot{H} X^{2}+2 \alpha H \dot{X} X+\frac{1}{2} \dot{\phi}^{2}+\epsilon \dot{\phi} X-V,
\end{aligned}
$$

где $H=\dot{a} / a$ - параметр Хаббла, и мы полагаем $N=1$ всюду далее в этом разделе. Интересующая нас величина имеет вид

$$
\rho+p=\epsilon \dot{\phi} X+\alpha \dot{H} X^{2}+2 \alpha H \dot{X} X+\kappa \dot{X}^{2}+\dot{\phi}^{2} .
$$

5) Наличия тахионной моды можно полностью избежать за счет тонкой настройки параметров $m_{1}^{2} / \kappa=\left(\epsilon^{2}-m_{1}^{2}\right) / \alpha$. В этом случае единственной потенциально опасной особенностью модели является наличие духа в ИК-области, т.е. при $q<q_{\mathrm{c}}$. Этот дух феноменологически приемлем даже при относительно больших $q_{\mathrm{c}}$, сильно превышающих параметр Хаббла [11].

6) Другая возможность состоит в том, что $X$ изменяется во времени достаточно медленно. Нам не потребуется прибегать к этой возможности. 
Наиболее важным для наших целей является первый член в этом выражении.

Уравнения движения для однородных полей имеют вид

$$
\begin{gathered}
-\kappa(\ddot{X}+3 H \dot{X})-\frac{1}{2} \kappa_{X} \dot{X}^{2}-\frac{3}{2} \alpha_{X} H^{2} X^{2}-3 \alpha H^{2} X+\epsilon \dot{\phi}=V_{X} \\
-(\ddot{\phi}+3 H \dot{\phi})-\epsilon(\dot{X}+3 H X)=V_{\phi} .
\end{gathered}
$$

Ниже мы полагаем, что $\alpha(X)$ и $\kappa(X)$ не являются быстро меняющимися функциями, так что

$$
\begin{aligned}
& \left|\alpha_{X}\right| \lesssim \frac{\alpha}{X}, \\
& \left|\kappa_{X}\right| \lesssim \frac{\kappa}{X} .
\end{aligned}
$$

Перейдем теперь к обсуждению режима медленного качения.

4.2. Медленное качение. Помимо обычного требования, заключающегося в том, что поля и параметр Хаббла изменяются медленно, определим особый режим медленного качения, представляющий интерес для наших целей. Будем рассматривать режим, при котором в левых частях полевых уравнений (33), (34) преобладают члены, пропорциональные $\epsilon$. В связи с этим в данном случае условия медленного качения не являются точно такими же, как в случае инфляции, управляемой скалярным инфлатоном (или в случае ускорения, управляемого скалярной квинтэссенцией). Одним из главных моментов является то, что в полевых уравнениях (33) имеются члены, не содержащие временны́х производных. Эти члены нежелательны, так что одним из наших условий является

$$
\alpha H^{2} X \ll \epsilon \dot{\phi}
$$

В силу соотношения (35) из этого условия следует, что оба члена в уравнении (33), не содержащие производных, малы. Здесь и далее при записи неравенства мы всегда будем иметь в виду абсолютные значения величин в левой и правой частях.

Еще одно нетривиальное условие медленного качения, обеспечивающее малость $\dot{\phi}$-члена в уравнении (34), имеет вид

$$
\dot{\phi} \ll \epsilon X .
$$

Другие условия очевидны:

$$
\begin{aligned}
\dot{X} & \ll H X, \\
\ddot{\phi} & \ll H \dot{\phi}, \\
\dot{H} & \ll H^{2} .
\end{aligned}
$$

Заметим, что в силу соотношений (35) и (36) член $\epsilon \dot{\phi}$ действительно доминирует над всеми другими членами в левой части уравнения (33), если выполняются условия (37) и (39). Наконец, потенциальный член доминирует при космологической эволюции, если

$$
V \gg \epsilon \dot{\phi} X
$$

4 Теоретическая и математическая физика, т. 149, № 3, 2006 г. 
Коль скоро эти условия медленного качения выполнены, полевые уравнения принимают простой вид

$$
\begin{gathered}
\epsilon \dot{\phi}=V_{X}, \\
3 H \epsilon X=-V_{\phi} .
\end{gathered}
$$

В режиме медленного качения первый член в правой части равенства (32) доминирует и имеет место соотношение

$$
\rho+p=X V_{X}
$$

Величина $\rho+p$ отрицательна при

$$
V_{X}<0
$$

(без потери общности мы полагаем, что $X>0$ ).

Уравнение (44) является алгебраическим, так что вся система уравнений медленного качения может показаться необычной. Чтобы лучше ее понять, поучительно взять потенциалы вида (5) и рассмотреть уравнение (43) как уравнение относительно $X$, решение которого имеет вид

$$
X=F(\dot{\phi}) \text {. }
$$

Оставляя в уравнении (34) только члены, пропорциональные $\epsilon$, запишем это уравнение следующим образом:

$$
\epsilon\left[F^{\prime}(\dot{\phi}) \ddot{\phi}+3 H F(\dot{\phi})\right]=-W_{\phi}
$$

Это уравнение можно понимать как полевое уравнение в скалярной теории поля с действием

$$
\int d^{4} x \sqrt{g}[K(\dot{\phi})-W(\phi)],
$$

где

$$
K(z)=\epsilon \int F(z) d z
$$

Тогда усеченное уравнение (44) является уравнением медленного качения для данной теории. Например, при

$$
U(X)=-\frac{M^{2}}{2} X^{2}
$$

из уравнения (43) имеем

$$
X=-\frac{\epsilon}{M^{2}} \dot{\phi},
$$

и уравнение (46) принимает вид

$$
\frac{\epsilon^{2}}{M^{2}}(\ddot{\phi}+3 H \dot{\phi})=+W_{\phi} .
$$


Это уравнение является обычным уравнением скалярного поля, но с перевернутым потенциалом. Другими словами, кинетический член в эффективном лагранжиане (47) имеет отрицательный знак. Это объясняет, почему в режиме медленного качения $\rho+p<0$.

Вернемся к общему случаю. Используя уравнения (43) и (44), можно переписать условия медленного качения в терминах потенциала $V(X, \phi)$ и его производных или, в специальном случае (5), в терминах $U(X), W(\phi)$ и их производных. Например, предполагая, что выполнено условие (41), из условия (39) находим, что

$$
\frac{1}{H \epsilon} W_{\phi \phi} U_{X} \ll W_{\phi} .
$$

Для потенциалов, удовлетворяющих соотношениям

$$
U_{X} \sim \frac{U}{X}, \quad W_{\phi} \sim \frac{W}{\phi}, \quad W_{\phi \phi} \sim \frac{W}{\phi^{2}}
$$

(например, для потенциалов, изменяющихся по степенному закону), снова воспользуемся уравнением (43) и из последнего неравенства получим, что

$$
W \gg U
$$

Таким образом, в уравнении Фридмана доминирует потенциал $W(\phi)$, так что

$$
H^{2}=\frac{8 \pi}{3 M_{\mathrm{Pl}}^{2}} W .
$$

Теперь ясно, что в режиме медленного качения плотность энергии действительно возрастает со временем. Предполагая без потери общности, что $X>0$, находим из уравнения (44), что $W_{\phi}<0$, в то время как из (43) и (45) следует, что $\dot{\phi}<0$. Это означает, что поле $\phi$ закатывается вверх по потенциалу; значение $W(\phi)$, а потому и энергия, возрастают.

Условие (40) дает

$$
\begin{aligned}
& \frac{1}{(\epsilon H)^{2}} U_{X X} W_{\phi \phi} \ll 1, \\
& \frac{\dot{H}}{H^{2}} \frac{1}{H \epsilon} U_{X X} W_{\phi} \ll U_{X} .
\end{aligned}
$$

Для потенциалов, удовлетворяющих соотношениям (50), воспользуемся уравнением (44) и получим, что соотношение (52) эквивалентно соотношению (41). Соотношения (49) и (51) говорят о том, что потенциалы являются достаточно плоскими.

Условие (37) дает соотношение несколько другого вида:

$$
W_{\phi} \ll \frac{\epsilon}{\alpha H} U_{X} .
$$

Аналогичным образом из условия (38) находим, что

$$
U_{X} \ll \frac{\epsilon}{H} W_{\phi} .
$$


Эти условия могут одновременно удовлетворяться только при

$$
\frac{\epsilon^{2}}{\alpha H^{2}} \gg 1
$$

Наконец, из условия (41) находим, что

$$
\frac{M_{\mathrm{Pl}}}{\epsilon W^{3 / 2}} W_{\phi} U_{X} \ll 1 .
$$

Чтобы показать, что все записанные выше условия действительно могут иметь место, сначала рассмотрим пример, когда $U(X)$ задается выражением (48). В этом случае эволюция поля $\phi$ и масштабного фактора соответствуют действию (47), причем

$$
K=-\frac{\epsilon^{2}}{2 M^{2}} \dot{\phi}^{2} .
$$

Таким образом, условия медленного качения (39)-(41), записанные в терминах поля

$$
\sigma=\frac{\epsilon}{M} \phi
$$

являются такими же, как и в теории инфляции; для потенциалов $W$, изменяющих ся по степенному закону, они дают $\sigma \gg M_{\mathrm{Pl}}$, т.е.

$$
\phi \gg \frac{M M_{\mathrm{Pl}}}{\epsilon} \text {. }
$$

Это, однако, не гарантирует выполнения условий (37) и (38), так что они должны быть наложены дополнительно к (56). Из условия (38) получаем, что

$$
M^{2} \ll \epsilon^{2},
$$

в то время как (37) дает

$$
\frac{M^{2}}{\alpha} \gg H^{2}
$$

В общем случае соотношения (56) и (58) удовлетворяются одновременно только на конечном интервале значений $\phi$; например, для $W=(1 / 2) \mu^{2} \phi^{2}$ этот интервал записывается как

$$
\frac{M M_{\mathrm{Pl}}}{\epsilon} \ll|\phi| \ll \frac{M M_{\mathrm{Pl}}}{\sqrt{\alpha} \mu}
$$

(заметим, что в силу (44) динамика медленного качения имеет место при $\phi<0$ для положительных $X$ ), что требует выполнения условия

$$
\mu^{2} \ll \frac{\epsilon^{2}}{\alpha}
$$

Чтобы закончить с этим примером, заметим, что условие (42) эквивалентно стандартным условиям медленного качения

$$
\dot{\sigma}^{2} \ll W
$$


и что мы действительно имеем

$$
\alpha X^{2} \ll M_{\mathrm{Pl}}^{2}
$$

(см. обсуждение после соотношения (31)) в силу неравенств (58) и (59). Таким образом, данный пример действительно представляет собой случай, в котором выполняются все наши требования.

В приведенном выше примере $U_{X X}<0$, так что масса (9) является отрицательной. В действительности в этом примере массы удовлетворяют соотношению (28), поэтому при больших импульсах не существует сверхсветовых мод и/или духов. Существование духов (29) при малых импульсах согласуется со знаком минус у величины $K(\dot{\phi})$ в выражении (47). Если добавить дополнительные члены в потенциал (48), при больших значениях пространственых импульсов мода (29) станет или субсветовой, или сверхсветовой.

Рассмотрим пример, для которого не обязательно выполняется условие $U_{X X}<0$ : зададим потенциалы как

$$
\begin{aligned}
& U(X)=\zeta^{2} X_{0}^{4} u\left(\frac{X}{X_{0}}\right), \\
& W(\phi)=\tau^{2} \phi^{4},
\end{aligned}
$$

где $X_{0}$ - некоторое типичное значение поля $X, u$ - гладкая функция порядка единицы, производные которой также являются функциями порядка единицы, а $\zeta$ и $\tau$ - константы взаимодействия (в этом примере, переопределив поля, мы положили $\alpha=1)$. Потенциал (60) выбран как функция четвертого порядка только для конкретности. Из уравнения (44) видно, что медленное качение возникает при $X \sim X_{0}$, если

$$
\phi \sim \frac{\epsilon X_{0}}{\tau M_{\mathrm{Pl}}} .
$$

Непосредственно проверяется, что все условия медленного качения выполняются, если параметры удовлетворяют соотношению

$$
\frac{M_{\mathrm{Pl}}^{4} \tau^{2} \zeta^{2}}{\epsilon^{4}} \ll 1
$$

а значение поля $X$ удовлетворяет условиям

$$
\begin{aligned}
& X_{0}^{2} \ll M_{\mathrm{Pl}}^{2} \frac{M_{\mathrm{Pl}}^{4} \tau^{2} \zeta^{2}}{\epsilon^{4}}, \\
& X_{0}^{2} \ll \frac{\epsilon^{2}}{\zeta^{2}} .
\end{aligned}
$$

Ясно, что такой выбор действительно можно осуществить.

\section{5. ВОЗМУЩЕНИЯ ВОКРУГ МЕДЛЕННО МЕНЯЮЩЕГОСЯ ФОНА}

Рассмотрим возмущения полей $B_{\mu}$ и $\Phi$ в режиме медленного качения. Предполагая, что все массы малы по сравнению с $M_{\mathrm{Pl}}$, мы пренебрежем смешиванием 
возмущений $B_{\mu}$ и $\Phi$ с возмущениями гравитационного поля ${ }^{7)}$. Для упрощения формул положим $\alpha$ и $\beta$ не зависящими от $X$; наши основные выводы остаются в силе и в общем случае.

Прежде чем продолжить, сделаем несколько замечаний относительно соотношений между массовыми членами, значением $\epsilon$ и параметром Хаббла. Во-первых, заметим, что неравенство (45), т.е. условие $X V_{X}<0$, обеспечивает положительность массового члена для векторных возмущений $b_{i}$, см. формулу (10). Во-вторых, из соотношений (53) и (54) и уравнения движения медленного качения (44) следует, что

$$
\epsilon^{2} \gg m_{1}^{2} \gg \alpha H^{2}
$$

где, как и ранее, $m_{1}^{2}=-U_{X} / X$. Аналогично из неравенства (51) следует, что $m_{\varphi}^{2} \equiv W_{\phi \phi}$ мало. Предполагая, что $U_{X X} \sim X^{-1} U_{X}$, мы, таким образом, приходим к рассмотрению случая, исследованного в п. 3.2 на фоне пространства Минковского.

В силу условий (55) и (61) параметр Хаббла мал по сравнению с $\epsilon / \sqrt{\alpha}, \epsilon / \sqrt{\kappa}$ и $m_{1} / \sqrt{\alpha}$. Поэтому расширение Вселенной не влияет на поперечные моды, которые имеют щель в спектре (22), а также на еще две моды со щелями (25) и (26). При заданном конформном импульсе $k$ одна мода становится тахионной, когда физический импульс сдвигается в красную область к значению $q_{\mathrm{c}}$, которое задается соотношением (18) и которое значительно больше, чем $H$. При дальнейшем сдвиге физического импульса в красную область эта мода входит в режим надгоризонта. Таким образом, нам надо изучить поведение этой моды в таком режиме ${ }^{8)}$.

Удобно работать с конформной метрикой

$$
d s^{2}=a^{2}(\eta)\left(d \eta^{2}-d \mathbf{x}^{2}\right)
$$

В этой метрике поля с возмущениями имеют вид

$$
B_{0}=a X+b_{0}, \quad B_{i}=b_{i}, \quad \Phi=\phi+\frac{1}{a} \chi,
$$

где $X$ и $\phi$ - фоновые поля. Уравнения для продольных компонент $b_{i}=\left(k_{i} / k\right) b_{L}, b_{0}$ и $\chi$ в конформном времени имеют вид ${ }^{9)}$

$$
\begin{array}{r}
-\kappa\left(b_{0}^{\prime \prime}-\frac{a^{\prime \prime}}{a} b_{0}+k^{2} b_{0}\right)-3 \alpha \frac{a^{\prime 2}}{a^{2}} b_{0}-a^{2} m_{0}^{2} b_{0}+2 i k \alpha \frac{a^{\prime}}{a} b_{\mathrm{L}}+\epsilon a\left(\chi^{\prime}-\frac{a^{\prime}}{a} \chi\right)=0, \\
-\alpha\left(b_{\mathrm{L}}^{\prime \prime}-\frac{a^{\prime \prime}}{a} b_{\mathrm{L}}-\frac{a^{\prime 2}}{a^{2}} b_{\mathrm{L}}+k^{2} b_{\mathrm{L}}\right)-a^{2} m_{1}^{2} b_{\mathrm{L}}-2 i k \alpha \frac{a^{\prime}}{a} b_{0}-i \epsilon k a \chi=0,
\end{array}
$$

7) Смешивание с возмущениями гравитационного поля на фоне пространства Минковского приводит к новым эффектам при малых по сравнению с рассмотренными здесь импульсах [13], [14]. Эти импульсы в нашем контексте лежат ниже хаббловского масштаба, так что в настоящей работе они не будут нас интересовать.

8) В случае $m_{0}^{2}=-m_{1}^{2}$, рассмотренном в конце п. 3.2 , при $q^{2}<m_{1}^{2} / \kappa$ не существует тахионных мод. Приведенный ниже анализ примени́м к духовой моде, соответствующей малому значению импульса и удовлетворяющей соотношению $\omega^{2}=q^{2}$ в пространстве-времени Минковского.

9) Мы не записываем здесь члены, пропорциональные $X^{\prime}$. Можно показать, что в режиме медленного качения эти члены являются малыми как для над-, так и для подгоризонтных мод. По той же причине мы пренебрегаем и зависимостью масс от времени. 


$$
-\chi^{\prime \prime}+\frac{a^{\prime \prime}}{a} \chi-k^{2} \chi-a^{2} m_{\varphi}^{2} \chi-\epsilon a\left(b_{0}^{\prime}+2 \frac{a^{\prime}}{a} b_{0}\right)+i \epsilon k a b_{\mathrm{L}}=0,
$$

где $k$ - конформный импульс, а штрих обозначает $\partial / \partial \eta$. Мы интересуемся медленными модами с малыми физическими импульсами,

$$
k / a \ll m_{0,1} / \sqrt{\alpha},
$$

и малыми по сравнению с $m_{0,1} / \sqrt{\alpha}$ производными по времени. Вспоминая, что $m_{0,1}^{2} \gg \alpha H^{2}$, пренебрежем первыми двумя членами в уравнении (62) и первым членом в уравнении (63). Тогда эти два уравнения становятся алгебраическими уравнениями относительно $b_{0}$ и $b_{\mathrm{L}}$, что дает

$$
\begin{aligned}
& b_{0}=\frac{\epsilon}{m_{0}^{2}}\left[\frac{1}{a} \chi^{\prime}-H \chi-\frac{2 \alpha H}{m_{1}^{2}}\left(\frac{k}{a}\right)^{2} \chi\right], \\
& b_{\mathrm{L}}=\frac{\epsilon}{m_{1}^{2}}\left[-i \frac{k}{a} \chi+\frac{2 i \alpha H}{m_{0}^{2}} \frac{k}{a}\left(\frac{1}{a} \chi^{\prime}-H \chi\right)\right],
\end{aligned}
$$

где $H=a^{\prime} / a^{2}$ - параметр Хаббла. Для вывода этих соотношений мы воспользовались условиями (61) и (65). Подставляя эти выражения в уравнение (64), получаем уравнение для $\chi$ :

$-\chi^{\prime \prime}+\frac{a^{\prime \prime}}{a} \chi-k^{2} \chi+\frac{\epsilon^{2}}{m_{0}^{2}}\left[-\chi^{\prime \prime}+\frac{a^{\prime \prime}}{a} \chi+\frac{m_{0}^{2}}{m_{1}^{2}} k^{2} \chi-a^{2} \frac{m_{0}^{2} m_{\varphi}^{2}}{\epsilon^{2}} \chi+2 \alpha \frac{k^{2}}{m_{1}^{2}}\left(H^{2}-\frac{1}{a} H^{\prime}\right) \chi\right]=0$.

Снова вспомнив соотношения (61), получим окончательное уравнение для мягкой моды:

$$
-\chi^{\prime \prime}+\frac{a^{\prime \prime}}{a} \chi+\frac{m_{0}^{2}}{m_{1}^{2}} k^{2} \chi-a^{2} \frac{m_{0}^{2} m_{\varphi}^{2}}{\epsilon^{2}} \chi=0 .
$$

Мы видим, что при $m_{\varphi}=0$ поле $\chi$ удовлетворяет уравнению для скалярного поля с тахионным дисперсионным соотношением (27), но теперь в расширяющейся Вселенной. Для не обращающихся в нуль $m_{\varphi}$ из соотношения (51) следует, что слагаемое с $m_{\varphi}^{2}$ мало по сравнению со вторым слагаемым (последнее имеет порядок $H^{2} a^{2} \chi$ ). Таким образом, решения уравнения (68) демонстрируют тахионное поведение при $k / a \gg H$ с дисперсионным соотношением (27).

С другой стороны, в режиме надгоризонта $k / a \ll H$ имеется обычная "постоянная" мода $\chi \propto a$ и убывающая мода (при не зависящем от времени $H$ последняя мода $\left.\chi \propto a^{-2}\right)$. Более точно, в пространстве-времени де Ситтера, где

$$
a=-\frac{1}{H \eta}
$$

"постоянная" мода имеет вид

$$
\chi=-\frac{c}{H \eta^{1-\delta}},
$$

где $c$ - малая амплитуда, а

$$
\delta=\frac{m_{\varphi}^{2} m_{0}^{2}}{3 \epsilon^{2} H^{2}} \ll 1
$$


Поэтому возмущение поля $\Phi$ почти не зависит от времени:

$$
\varphi \equiv \frac{1}{a} \chi=c \eta^{\delta} .
$$

Из соотношения (66) следует, что для "постоянной” моды

$$
b_{0}=\frac{\epsilon H}{m_{0}^{2}} \delta \chi .
$$

Это означает, что физическая временна́я компонента возмущения векторного поля также медленно изменяется со временем:

$$
\frac{b_{0}}{a} \sim c \frac{m_{\varphi}^{2}}{\epsilon H} \eta^{\delta} .
$$

С другой стороны, из соотношения (67) следует, что $b_{\mathrm{L}}$ не зависит от времени для "постоянной" моды, так что физическая пространственная компонента векторного поля $B_{i} / a$ убывает как $a^{-1}$.

Таким образом, можно сделать вывод, что в режиме надгоризонта тахионная мода больше не представляет проблемы, поскольку она становится практически вмороженной. Эта мода имеет конечное время для развития в расширяющейся Вселенной.

\section{6. ЗАКЛЮЧЕНИЕ}

Нет необходимости говорить, что для того чтобы наша модель стала жизнеспособным кандидатом на описание инфляции или современной космологической эволюции, ей требуется немалая тонкая настройка. В частности, отношение $\epsilon^{2} /\left(\alpha H^{2}\right)$ в режиме медленного качения должно быть достаточно, но не чрезмерно большим. Действительно, начиная с того момента времени, когда одна из мод становится тахионной (это происходит при $k / a=\epsilon / \sqrt{\alpha}$ ), и до того момента времени, когда эта мода выходит за горизонт (при $k / a \sim H$ ), фактор, определяющий рост этой моды, имеет вид

$$
\exp \left(N_{\text {growth }}\right) \sim \exp \left(\frac{1}{H} \int_{H}^{\sqrt{\epsilon^{2} / \alpha}} \omega(q) \frac{d q}{q}\right) .
$$

Интеграл в этом выражении насыщается при $q \sim q_{\text {c }}$ и, используя соотношение (19), мы можем сделать оценку

$$
N_{\text {growth }}=\text { const } \cdot \sqrt{\frac{\kappa}{\alpha}} \cdot \sqrt{\frac{\epsilon^{2}}{\alpha H^{2}}} .
$$

Для того чтобы фактор, определяющий рост, не был огромным, следует провести или тонкую настройку величины $\kappa$, так чтобы она была много меньше $\alpha$, или тонкую настройку величины $\epsilon^{2} /\left(\alpha H^{2}\right)$, так чтобы его значение не было слишком большим, или и то, и другое ${ }^{10)}$. Грубо говоря, плотность энергии, соответствующая неоднородным модам, составляет малую долю от плотности энергии фона, только если

$$
\frac{\sqrt{\kappa}}{\alpha} \frac{\epsilon}{H} \ll \ln \frac{M_{\mathrm{Pl}}}{\epsilon} .
$$

10) Как было отмечено выше, другая возможность заключается в тонкой настройке параметров $m_{1}^{2} / \kappa=\left(\epsilon^{2}-m_{1}^{2}\right) / \alpha$. В этом случае тахион вообще не существует. 
Было бы интересно понять, могут ли эта, а также много других тонких настроек возникать автоматически.

Представленная в настоящей работе модель является почти наверняка не самой привлекательной фантомной теорией, безопасной в УФ-диапазоне. Мы скорее пытались показать, что такая теория вообще возможна. Поэтому мы не делали попыток исследовать целый ряд важных проблем: может ли режим медленного качения быть космологическим аттрактором, имеется ли в инфляционном контексте естественный способ выхода из такого режима, может ли в контексте современной Вселенной тахионная неустойчивость на длинных волнах иметь интересные следствия и т.д. Еще до того, как поставить эти вопросы, было бы желательно понять, какие свойства нашей модели являются общими, а какие зависят от модели. В частности, было было бы интересно выяснить, можно ли избежать тахионов, которые существуют в нашей модели для довольно широкого диапазона значений пространственных импульсов выше $H$, оставив при этом менее опасные ИК-духи.

Благодарности. Автор признателен Д. Горбунову, Д. Левкову, М. Либанову, С. Сибирякову и П. Тинякову за ценные обсуждения, а также С. Дубовскому за полезную переписку. Настоящая работа выполнена при частичной финансовой поддержке РФФИ (грант № 05-02-17363-а).

\section{Список литературы}

[1] А.А. Логунов, М.А. Мествиришвили, Релятивистская теория гравитации, Наука, М., 1989; А.А. Логунов, Теория гравитационного поля, Наука, М., 2001; gr-qc/0210005; С. С. Герштейн, А. А. Логунов, М. А. Мествиришвили, Н. П. Ткаченко, ЭЧАЯ, 36 (2005), 1003; C. Charmousis, R. Gregory, V. A. Rubakov, Phys. Rev. D, 62 (2000), 067505; hep-th/9912160; R. Gregory, V. A. Rubakov, S. M. Sibiryakov, Phys. Rev. Lett., 84 (2000), 5928; hep-th/0002072; I. I. Kogan, S. Mouslopoulos, A. Papazoglou, G. G. Ross, J. Santiago, Nucl. Phys. B, 584 (2000), 313; hep-ph/9912552; I. I. Kogan, G. G. Ross, Phys. Lett. B, 485 (2000), 255; hep-th/0003074; D. J. H. Chung, K. Freese, Phys. Rev. D, 61 (2000), 023511; hep-ph/9906542; K. Freese, M. Lewis, Phys. Lett. B, 540 (2002), 1; astro-ph/0201229; I. T. Drummond, Phys. Rev. D, 63 (2001), 043503; astro-ph/0008234; N. Arkani-Hamed, H. C. Cheng, M. A. Luty, S. Mukohyama, JHEP, 05 (2004), 074; hep-th/0312099; J.W. Moffat, Modified gravitational theory as an alternative to dark energy and dark matter, astro-ph/0403266; V.A. Rubakov, Lorentz-violating graviton masses: Getting around ghosts, low strong coupling scale and VDVZ discontinuity, hep-th/0407104; S. L. Dubovsky, JHEP, 10 (2004), 076; hep-th/0409124; S. L. Dubovsky, P. G. Tinyakov, I. I. Tkachev, Phys. Rev. D, 72 (2005), 084011; hep-th/0504067; J. D. Bekenstein, Modified gravity vs dark matter: Relativistic theory for MOND, PoS, JHW2004 (2005), 012; astro-ph/0412652.

[2] G. R. Dvali, G. Gabadadze, M. Porrati, Phys. Lett. B, 485 (2000), 208; hep-th/0005016.

[3] R. R. Caldwell, Phys. Lett. B, 545 (2002), 23; astro-ph/9908168; A. E. Schulz, M. J. White, Phys. Rev. D, 64 (2001), 043514; astro-ph/0104112; R. R. Caldwell, M. Kamionkowski, N. N. Weinberg, Phys. Rev. Lett., 91 (2003), 071301; astro-ph/0302506; J. G. Hao, X.Z. Li, Phys. Rev. D, 67 (2003), 107303; gr-qc/0302100; G.W. Gibbons, Phantom matter and the cosmological constant, hep-th/0302199; S. Nojiri, S. D. Odintsov, Phys. Lett. B, 562 (2003), 147; hep-th/0303117; P. Singh, M. Sami, 
N. Dadhich, Phys. Rev. D, 68 (2003), 023522; hep-th/0305110; M. P. Dabrowski, T. Stachowiak, M. Szydlowski, Phys. Rev. D, 68 (2003), 103519; hep-th/0307128; J. G. Hao, X.Z. Li, Phys. Rev. D, 70 (2004), 043529; astro-ph/0309746; V. B. Johri, Phys. Rev. D, 70 (2004), 041303; astro-ph/0311293; U. Alam, V. Sahni, T. D. Saini, A. A. Starobinsky, Mon. Not. Roy. Astron. Soc., 354 (2004), 275; astro-ph/0311364; M. Sami, A. Toporensky, Mod. Phys. Lett. A, 19 (2004), 1509; gr-qc/0312009; B. Feng, X. L. Wang, X. M. Zhang, Phys. Lett. B, 607 (2005), 35; astro-ph/0404224; Z. K. Guo, Y.S. Piao, X. M. Zhang, Y.Z. Zhang, Phys. Lett. B, 608 (2005), 177; astro-ph/0410654; G. B. Zhao, J. Q. Xia, B. Feng, X. Zhang, Probing dynamics of dark energy with supernova, galaxy clustering and the three-year Wilkinson Microwave Anisotropy Probe (WMAP) observations, astro-ph/0603621.

[4] B. Boisseau, G. Esposito-Farese, D. Polarski, A. A. Starobinsky, Phys. Rev. Lett., 85 (2000), 2236; gr-qc/0001066; A. Riazuelo, J.-P. Uzan, Phys. Rev. D, 66 (2002), 023525; astro-ph/0107386; D.F. Torres, Phys. Rev. D, 66 (2002), 043522; astro-ph/0204504; L. Perivolaropoulos, JCAP, 0510 (2005), 001; astro-ph/0504582; M.X. Luo, Q. P. Su, Phys. Lett. B, 626 (2005), 7; astro-ph/0506093; J. Martin, C. Schimd, J.-P. Uzan, Phys. Rev. Lett., 96 (2006), 061303; astro-ph/0510208.

[5] S. M. Carroll, A. De Felice, M. Trodden, Phys. Rev. D, 71 (2005), 023525; astro-ph/0408081.

[6] S. Dubovsky, T. Gregoire, A. Nicolis, R. Rattazzi, JHEP, 03 (2006), 025; hep-th/0512260.

[7] A. Vikman, Phys. Rev. D, 71 (2005), 023515; astro-ph/0407107; R. V. Buniy, S. D. H. Hsu, Phys. Lett. B, 632 (2006), 543; hep-th/0502203.

[8] W. Hu, Phys. Rev. D, 71 (2005), 047301; astro-ph/0410680.

[9] A. Nicolis, R. Rattazzi, JHEP, 06 (2004), 059; hep-th/0404159.

[10] V. Sahni, Y. Shtanov, JCAP, 0311 (2003), 014; astro-ph/0202346; A. Lue, G. D. Starkman, Phys. Rev. D, 70 (2004), 101501; astro-ph/0408246.

[11] J. M. Cline, S. Jeon, G. D. Moore, Phys. Rev. D, 70 (2004), 043543; hep-ph/0311312.

[12] A. Adams, N. Arkani-Hamed, S. Dubovsky, A. Nicolis, R. Rattazzi, Causality, analyticity and an IR obstruction to UV completion, hep-th/0602178.

[13] B. M. Gripaios, JHEP, 10 (2004), 069; hep-th/0408127.

[14] M. V. Libanov, V. A. Rubakov, JHEP, 08 (2005), 001; hep-th/0505231.

[15] S. M. Carroll, E. A. Lim, Phys. Rev. D, 70 (2004), 123525; hep-th/0407149.

Поступила в редакцию 10.05.2006 